\section{Application of alanine dosimetry \\ in dose assessment \\ for ocular melanoma patients \\ undergoing proton radiotherapy \\ - preliminary results}

\author{
Gabriela Mierzwińska, \\ Magdalena Kłodowska, \\ Barbara Michalec, \\ Anna Pedracka, \\ Marzena Rydygier, \\ Jan Swakoń, \\ Michael P. R. Waligórski
}

\begin{abstract}
Basing on alanine solid state/electron paramagnetic resonance (EPR) dosimetry, a supplementary method of cumulatively recording the therapeutic dose received by ocular cancer patients undergoing fractionated proton radiotherapy is proposed. By applying alanine dosimetry during the delivery of consecutive fractions, the dose received within each fraction can be read out by EPR spectrometry and a final permanent cumulative record of the total dose delivered obtained. The dose response of the alanine detector was found to be practically independent on its position within the extended proton Bragg peak region. Dose measurements based on entrance dose recorded in proton beams individually formed for each patient are presented. The described method will be applied as a complementary Quality Assurance procedure for patients undergoing proton radiotherapy at the Institute of Nuclear Physics, Polish Academy of Sciences, Krakow, Poland (IFJ PAN).
\end{abstract}

Key words: alanine $\bullet$ EPR dosimetry $\bullet$ proton beam $\bullet$ proton radiotherapy $\bullet$ Quality Assurance (QA)

G. Mierzwińska ${ }^{\bowtie}$, M. Kłodowska, B. Michalec, A. Pędracka, M. Rydygier, J. Swakoń

The Henryk Niewodniczański Institute of Nuclear Physics of the Polish Academy of Sciences,

152 Radzikowskiego Str., 31-342 Krakow, Poland, Tel.: +48 12662 8327, Fax: +48 12662 0866,

E-mail: gabriela.mierzwinska@ifj.edu.pl

M. P. R. Waligórski

The Henryk Niewodniczański Institute of Nuclear Physics of the Polish Academy of Sciences, 152 Radzikowskiego Str., 31-342 Krakow, Poland and Centre of Oncology, Maria Skłodowska-Curie Memorial Institute, Krakow Branch,

11 Garncarska Str., 31-115 Krakow, Poland

Received: 29 September 2014

Accepted: 20 May 2015

\section{Introduction}

IFJ PAN is the first Polish proton radiotherapy facility where ocular melanoma patients are treated using a cyclotron-produced $60 \mathrm{MeV}$ proton beam. Until now, over 70 patients have been treated in this facility. To treat ocular choroidal melanoma, the total prescribed dose delivered to the planning target volume (PTV) of 60 CGE (cobalt grey equivalent) is recommended, in four consecutive daily fractions of 15 CGE each, where CGE accounts for the relative biological effectiveness (RBE) of protons in the eye tissue. The International Commission on Radiation Units and Measurements - ICRU - recommended RBE value for protons under all clinical conditions is 1.1 [1], thus the physical dose delivered to the PTV to treat ocular melanoma is 15 CGE/1.1 = 13.64 Gy per fraction. In the presently used treatment setup, during patient irradiation, dose delivery is controlled by a system based on transmission ion chambers only - therefore, an independent method of verifying the dose delivered to the patient's PTV is highly desirable. Alanine dosimetry appears to be a good candidate, as it is a passive dosimeter exhibiting a linear dose response dependence in the therapeutic dose range and a stable, re-readable ESR signal $[2,3]$. By successively reading out via ESR spectrometry (without destroying the free-radical signal) the dose accumulated in consecutive fractional exposures, both individual dose per fraction and its final accumulated value, can be read out and recorded for each patient. Along these principles, we plan to develop a QA procedure to verify fractional and cu- 
mulated dose delivered to the patient's PTV. During patient treatment, the alanine detector should not be placed directly in the beam delivered to the patient, as it would then affect the dose delivered to the PTV. We therefore decided to place the alanine detectors on the inner side of the individual collimator assembly and seek suitable correction factors to relate the value of dose readout by the alanine detectors to the value of dose applied to the PTV, accounting for the dependence of such factors on the beam-forming elements individually tailored for each patient. The aim of this work is to develop and evaluate such correction factors by establishing the correlation between the entrance dose value (as registered by alanine detectors placed inside the individual proton beamforming collimators) and the value of dose delivered to the PTV of individual patients.

\section{Materials and methods}

Alanine is a radiosensitive material in which ionizing radiation generates stable free radicals. The concentration of these free radicals can be measured quantitatively by electron paramagnetic resonance (EPR) $[2,3]$. In our study, pellet-shaped alanine detectors, of $3 \mathrm{~mm}$ thickness and $4.8 \mathrm{~mm}$ diameter purchased from Synergy Health (Radeberg, Germany), were used As given by the manufacturer, these detectors are composed of $96 \mathrm{wt} \%$ alanine and $4 \mathrm{wt} \%$ binding material (polyethylene and additives). Some of the main properties of these alanine detectors have already been investigated $[4,5]$. Linear calibration

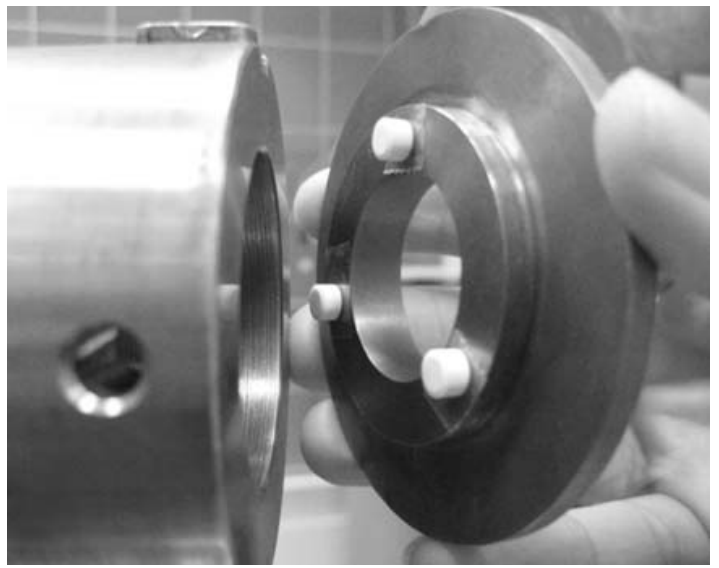

Fig. 1. Placement of the alanine detectors inside the final collimator. curves were established over the range 1-100 Gy, relating the EPR signal of alanine and proton beam dose, measured with a reference Markus-type ionization chamber with overall relative uncertainties of: $\pm 5.18 \%$ at $5 \mathrm{~Gy}, \pm 4.80 \%$ at $10 \mathrm{~Gy}$, and $\pm 4.70 \%$ at $20 \mathrm{~Gy}[4,5]$.

For the purpose of this study, three alanine detectors were inserted inside the $\varnothing 25 \mathrm{~mm}$ circular collimator (Fig. 1) located at the end of the snout, which forms the final part of the beam delivery system (Fig. 2). The $\varnothing 25 \mathrm{~mm}$ circular collimator illustrated in Fig. 1 is typically used for QA and dosimetry measurements. Therapeutic dose delivery requires individually shaped collimators of irregular shape that reproduces the projection of the patient's ocular PTV on a plane perpendicular to the proton beam axis.

The irradiated alanine detectors were read out using a Bruker ESP 300 spectrometer setup as follows: modulation amplitude $9.77 \mathrm{G}$, microwave power $7.93 \mathrm{~mW}$, modulation frequency $100 \mathrm{kHz}$, conversion time $20.48 \mathrm{~ms}$, time constant $327.68 \mathrm{~ms}$, receiver gain $2 \cdot 10^{4}$ and magnetic field resolution 1024 points. In order to improve the signal-to-noise ratio, the cumulative readout mode was used, with each scan being repeated three times. Within the adopted readout procedure, each detector was read out five times, with the sample being repositioned in the spectrometer cavity between readouts. Dose evaluation procedure was conformed with the requirements of ISO [6]. In each exposure of alanine detectors described below, three alanine detectors placed inside the collimator were irradiated. The final result is given as an average value of the readout of these three detectors.

Alanine detectors were irradiated in the $60 \mathrm{MeV}$ proton beam, generated by the AIC- 144 cyclotron and delivered to the therapeutic room. The beam is then individually formed and monitored by devices mounted on the optical bench, as illustrated in Fig. 2.

The dose-forming system consists of a range shifter and range modulator, which shape the extended Bragg peak of the pristine beam (proton depth-dose distribution profile, corresponding to $28.9 \mathrm{~mm}$ range in water for a $60 \mathrm{MeV}$ beam) with respect to the depth, dimensions and location of the tumor, as determined by the treatment planning system. The range shifter is a PMMA (poly (methyl methacrylate)) wheel of gradually varying thickness, which adjusts the proton beam range to the distal edge of the tumor volume. The range modulator is

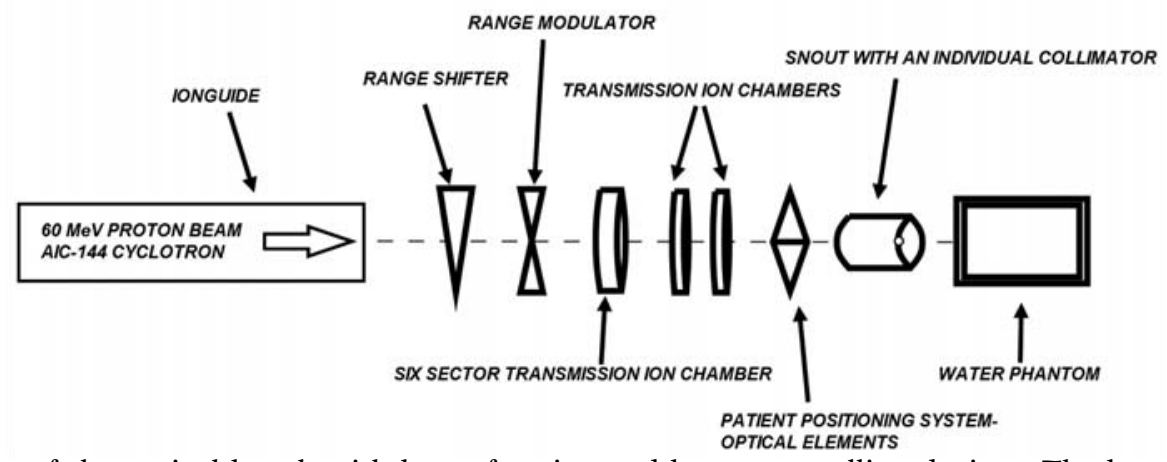

Fig. 2. Schematics of the optical bench with beam-forming and beam-controlling devices. The beam enters on the left-hand side of this figure. 


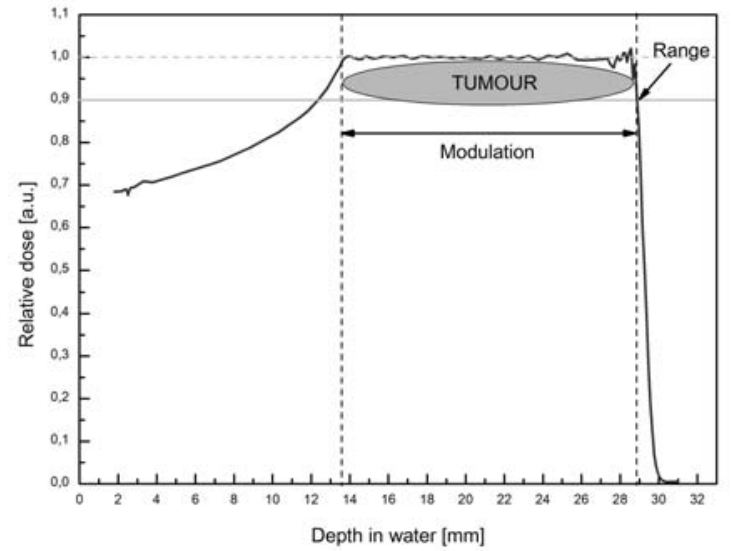

Fig. 3. A depth-dose distribution profile (spread-out Bragg peak) of specified range and modulation (in this case-partial modulation).

an individually made PMMA propeller, which serves to broaden (or 'modulate') the narrow Bragg peak into the so-called spread-out Bragg peak (SOBP), a uniform depth-dose profile, matching the thickness of the PTV along the beam axis. In summary, application of the range shifter and range modulator results in achieving the required dose distribution of depth and modulation, individually adjusted for each patient, as illustrated in Fig. 3.

The six-sector transmission chamber and two transmission ion chambers (PTW 7862 - PTW Freiburg, Freiburg in Breisgau, Germany) represented in Fig. 2, perform on-line beam intensity measurements. An in-house-developed dedicated software is used to control and transfer data, and monitor the performance of the beam forming and monitoring equipment [7]. In order to calibrate the dose delivered within each fraction using the reference Markus chamber, the operator sets the limiting value of charge, which when accumulated by the transmission chambers, triggers beam cut-off. During patient irradiation, beam delivery and beam cut-off are controlled only by the transmission chambers and the pre-set cut-off value of accumulated charge.

In this study, we performed alanine dosimetry during a routine control dosimetry session typically preceding patient irradiation, always by a dose 13.64 Gy per fraction, but with individual setup for each patient. In all, alanine dosimetry was performed within individual setups prepared for 37 different choroidal melanoma patients. For each patient, an individual set of beam forming devices (range shifter and range modulator) was prepared. In five cases, these individual patient setups were identical.

The dose delivered to the PTV was measured in an in-house manufactured water phantom using a PTW 23343 Markus plane-parallel ionization chamber and dedicated Ergen and PTW 10001 Unidos electrometers. After measuring the depth-dose within the spread-out Bragg peak (SOBP) according to the IAEA TRS-398 protocol, the Markus chamber was placed at mid-depth of the SOBP. This point, which was also the isocenter of the patient irradiation facility, was considered as the reference point for dosimetry measurements [8].

\section{Results and discussion}

Monitor chamber settings depend on the beam-forming elements. Due to beam absorption and scattering in the range shifter and range modulator material (PMMA), it is their effective thickness (represented in terms of corresponding water layer thickness) that determines the value (in MU - Monitor Units) of cut-off charge that controls the delivery of the required dose $(13.64 \mathrm{~Gy})$ to the isocenter. Figure 4 presents the relation between the charge of the monitor chamber and the effective thickness of beam-forming elements.

Two cases have to be considered: when the broadening of the SOBP covers the entire proton beam range (so-called full modulation) and when the SOBP is only partly broadened (partial modulation). In the case of partial modulation, the monitor charge increases with growing thickness of absorbing material according to the correlation formula given in Fig. 4 (for 17 patient setups). In the case of full SOBP broadening (for 20 patient setups), another set of correlation parameters best fits the data, as shown in Fig. 5.

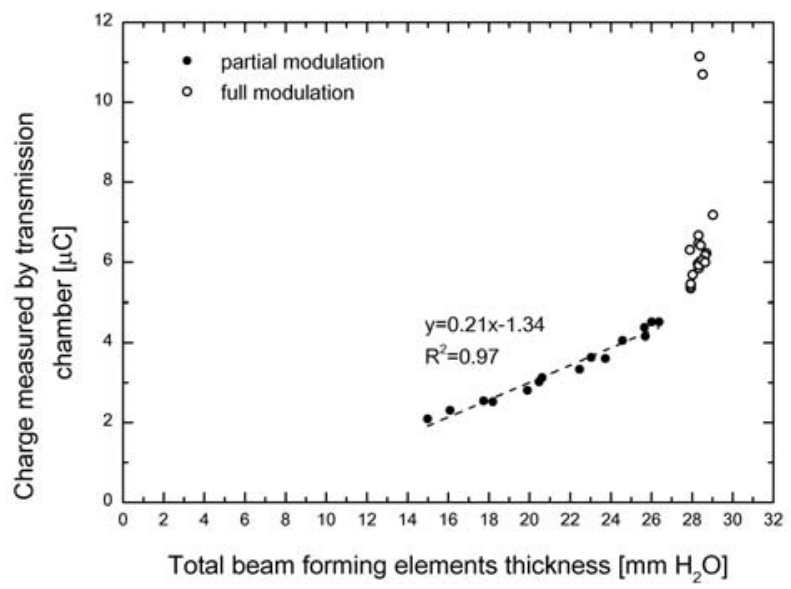

Fig. 4. Charge measured by the transmission chamber (in Monitor Units referred to the isocenter) vs. water equivalent thickness of the beam-forming elements. The regression equation in this figure is $\mathrm{y}[\mu \mathrm{C}]=0.21$ $\left[\mu \mathrm{C} / \mathrm{mm} \mathrm{H}_{2} \mathrm{O}\right] \times\left[\mathrm{mm} \mathrm{H}_{2} \mathrm{O}\right]-1.34[\mu \mathrm{C}]$.

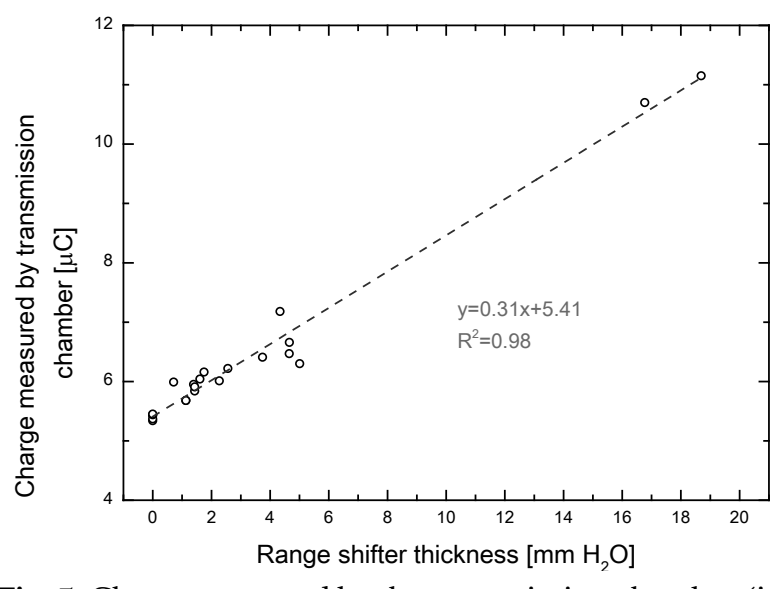

Fig. 5. Charge measured by the transmission chamber (in Monitor Units referred to the isocenter) vs. water equivalent thickness of the beam-forming elements, in the case of full spread-out Bragg peak broadening. The regression equation in this figure is $\mathrm{y}[\mu \mathrm{C}]=0.31\left[\mu \mathrm{C} / \mathrm{mm} \mathrm{H}_{2} \mathrm{O}\right] \times$ $\left[\mathrm{mm} \mathrm{H}_{2} \mathrm{O}\right]+5.41[\mu \mathrm{C}]$. 


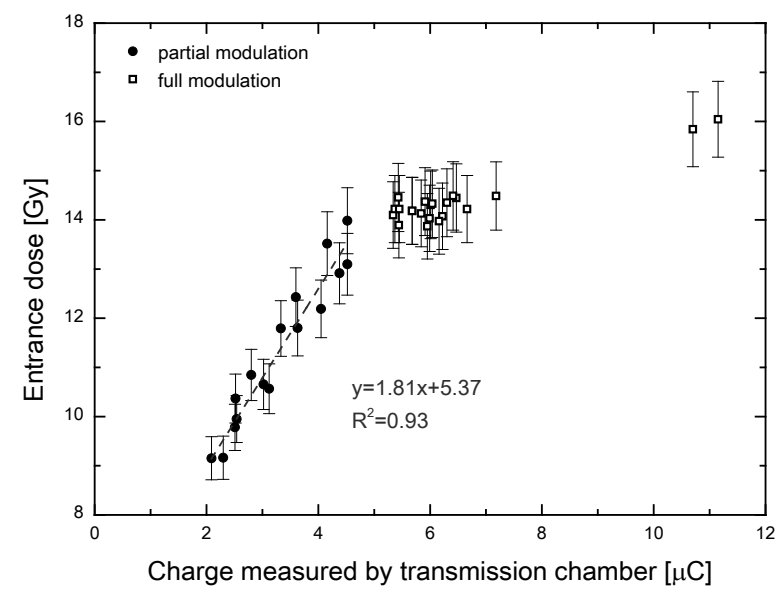

Fig. 6. Relation between the entrance dose measured with alanine detectors and transmission chamber Monitor Units-metered dose at the isocenter. The regression equation in this figure is $\mathrm{y}[\mathrm{Gy}]=1.81[\mathrm{~Gy} / \mu \mathrm{C}] \times[\mu \mathrm{C}]$ $+5.37[\mathrm{~Gy}]$.

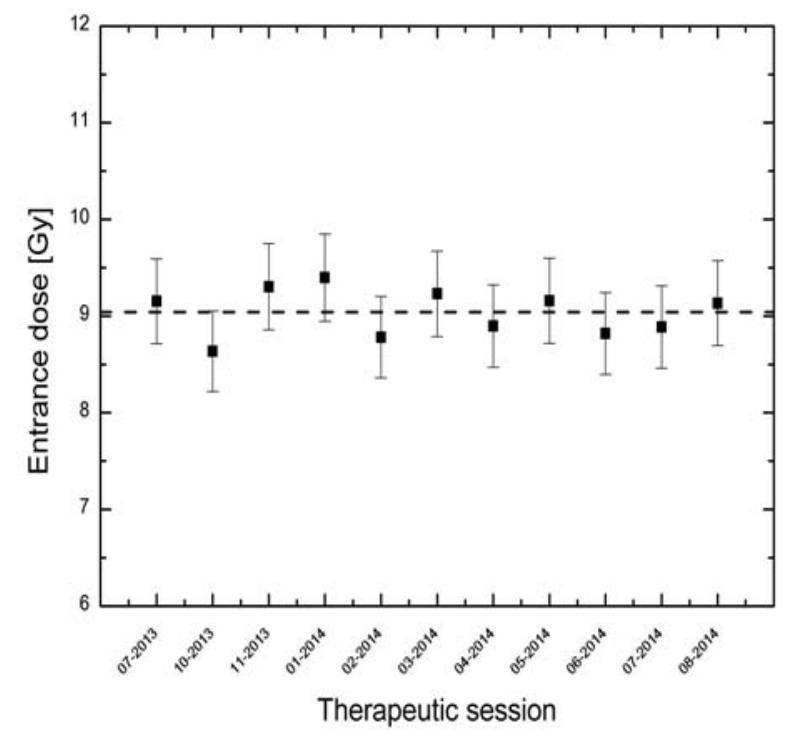

Fig. 7. Entrance dose measurements in a routine QA procedure delivering $13.64 \mathrm{~Gy}$ at the isocenter, for a full range spread-out Bragg peak of $15.2 \mathrm{~mm}$ modulation in water.

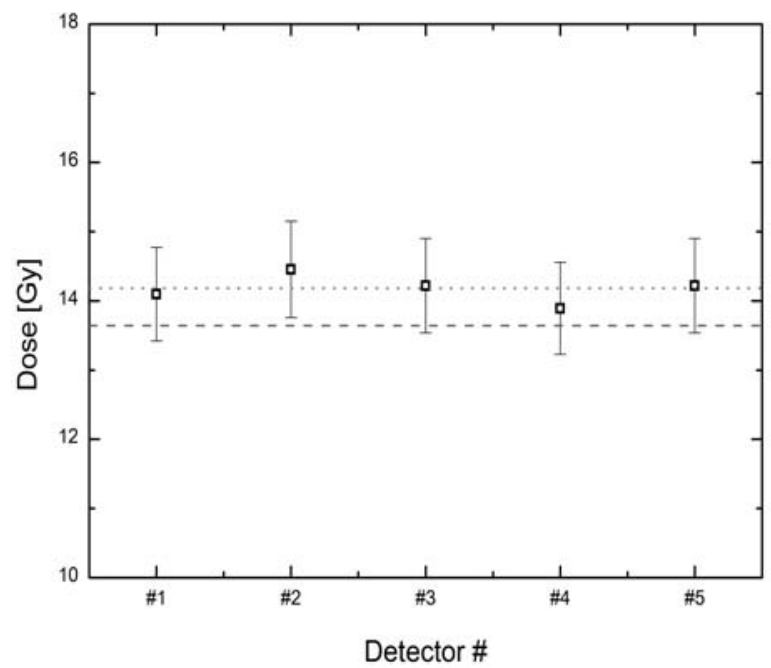

Fig. 8. Entrance dose measurements for full range modulation setup. Dashed line marks the isocenter dose level $(13.64 \mathrm{~Gy})$, the dotted line represents the average of five data points measured by alanine detectors (14.18 Gy).
As pointed out earlier, all results refer to the dose of $13.64 \mathrm{~Gy}$ delivered at the isocenter, hence the charge measured by the monitor chamber is specified by the individual set of beam-forming elements. Basing on correlation formulae shown in Figs. 4 and 5 , we combined the entrance dose, as measured by the alanine detectors, with the MU-metered monitor charge (now, independently of equivalent thickness of beam-forming elements). The result is given in Fig. 6 . In the case of partial SOBP broadening, one may approximate this relationship by a linear dependence, given by the formula in Fig. 6. Such analysis for the case of full modulation is less straightforward. One should note that since these measurements are based on actual patient setups, they cover the entire region of interest.

The patient-specific setup is accompanied by a routine QA procedure performed on a daily basis during the therapeutic session. This procedure uses the $\varnothing 25 \mathrm{~mm}$ circular collimator, involves a SOBP measurement and dose calibration using a standard range modulator, which produces a full range SOBP of $15.2 \mathrm{~mm}$ modulation in water. Within this setup procedure, we also irradiated alanine detectors. The results, for 11 such measurements are shown in Fig. 7, demonstrating that alanine dosimetry may also be included in such a QA procedure. The average readout in Fig. 7 is $9.04 \pm 0.21 \mathrm{~Gy}$.

The SOBP is considered a region of uniform depth-dose dependence over the whole desired depth obtained with range shift and modulation. In the case of full SOBP broadening, one would expect the entrance dose to be equal to the dose at isocenter. However, higher entrance dose levels have been found by alanine dosimetry. For instance, if a range modulator designed for full modulation over the whole $60 \mathrm{MeV}$ proton range was used, the entrance dose, as measured by alanine detectors, was on average higher by $4 \%$, than that measured at the isocenter (Fig. 8). Such measurements were performed preceding five therapeutic sessions. The dotted line in Fig. 8 represents the average value of the obtained results, and the dashed line indicates the 13.64 Gy level. The difference between doses at entrance and at the isocenter is as much as $17.7 \%$ for a SOBP of $10 \mathrm{~mm}$ range and modulation in water. Such differences are related to the position of the alanine detectors inside the collimator snout, which is at a distance of $10 \mathrm{~cm}$ from the position of the Markus chamber used for reference dosimetry.

\section{Conclusions}

Through our measurements, we have shown the potential - applicability of an alanine-based dose monitoring system that can be implemented as an independent tool for controlling the dose delivered to the PTV in ocular tumor treatment. As reported, measurement of the entrance dose with alanine detectors may supplement the present on-site beam control system which is calibrated daily to deliver the required dose per fraction and corrected with respect to the individual set of beam-forming devices. The 
alanine-based method, after further development, will serve to verify the delivery of therapeutic dose.

According to several recommendations, the uncertainty of therapeutic dose delivery to the target volume in radiotherapy should not exceed 5\% [9, 10]. Although our above-described method meets this requirement, more attention should be paid to increase the accuracy of alanine dosimetry developed at the IFJ PAN. This would further help to improve the QA of the complex proton radiotherapy procedure to treat choroidal melanoma and, in future, other sites in cancer patients.

\section{References}

1. ICRU. (2007). Prescribing, recording, and reporting proton-beam therapy. International Commission on Radiation Units and Measurements, Bethesda, Maryland. (ICRU Report 78).

2. Ahlers, F. J., \& Schneider, C. C. J. (1991). Alanine ESR dosimetry: an assessment of peak-to-peak evaluation. Radiat. Prot. Dosim., 37, 117-122.

3. Schauer, D. A., Iwasaki, A., Romanyukha, A. A., Swartz, H. M., \& Onori, S. (2006). Electron paramag- netic resonance (EPR) in medical dosimetry. Radiat. Meas., 41(Suppl.), S117-S123.

4. Michalec, B., Mierzwińska, G., Sowa, U., Ptaszkiewicz, M., Nowak, T., \& Swakoń, J. (2012). Alanine dosimetry of $60 \mathrm{MeV}$ proton beam - preliminary results. Nukleonika, 57, 503-506.

5. Mierzwinska, G., Michalec, B., Oglodek, I., Petelenz, B., \& Waligorski, M. P. R. (2014). Alanine/EPR dosimetry as a potential tool for quality assurance in proton beam radiotherapy. Rom. Rep. Phys., 66, 54-60.

6. International Standard ISO/WD 15566.1. (1998). Practice for use of alanine-EPR dosimetry system (1998). International Organization of Standardization

7. Michalec, B., Swakon, J., Sowa, U., Ptaszkiewicz, M., Cywicka-Jakiel, T., \& Olko, P. (2010). Proton radiotherapy facility for ocular tumours at the IFJ PAN in Krakow, Poland. Appl. Radiat. Isot., 68, 738-742.

8. Absorbed dose determination in external beam radiotherapy. (2000). Vienna: International Atomic Energy Agency. (Technical Reports Series no. 398).

9. ICRU. (2006). Measurement quality assurance for ionizing radiation dosimetry. International Commission on Radiation Units and Measurements, Bethesda, Maryland. (ICRU Report 76).

10. Podgorsak, E. B. (Ed.). (2005). Radiation oncology physics: a handbook for teachers and students. Vienna: International Atomic Energy Agency. 